\title{
Conditional survival in patients with gallbladder cancer
}

\author{
Yi-Jun Kim and Kyubo Kim*
}

\begin{abstract}
Background: Conditional survival (CS) has been established as a clinically relevant prognostic factor for cancer survivors, and the CS in gallbladder (GB) cancer has not yet been fully evaluated. In this study, we evaluated the cancerspecific CS rate and cancer-specific survival (CSS) rate in patients with GB cancer at multiple time points and investigated prognostic factors which affect cancer-specific CS rate to provide more accurate survival information.

Methods: Between 2004 and 2013, a total of 9760 patients with GB cancer were identified from the Surveillance, Epidemiology, and End Results (SEER) data. The 3-year cancer-specific CS rate was calculated using the covariateadjusted survival function in the Cox model for each year since diagnosis, and the results were analyzed together with the adjusted CSS rates at the same time points. Cox proportional hazards regression was performed to ascertain the individual contribution of factors associated with CSS rate at diagnosis and cancer-specific CS rates at 1, 3, and 5 years after diagnosis.

Results: The adjusted 5-year CSS rate was 26.1\%. The adjusted 3-year cancer-specific CS rates at 1, 2, 3, 4, and 5 years after diagnosis were $55.5,72.2,81.5,86.8$, and $90.5 \%$, respectively. At the time of diagnosis, age, race, histology, grade, $\mathrm{T}, \mathrm{N}$, and $\mathrm{M}$ categories, surgery, radiotherapy, insurance status, and marriage status were significant prognostic factors of CSS. Five years after diagnosis, however, T and $\mathrm{M}$ categories were significant prognostic factors for survivors $(P=0.007$ and $P=0.009$, respectively), whereas surgery and radiotherapy were not.

Conclusions: T and $M$ categories were significant prognostic factors even 5 years after the initial diagnosis, whereas local treatments at the time of diagnosis were not, suggesting that patients with GB cancer at high risks might need further adjuvant therapy after primary treatments. The combined analysis of CSS and cancer-specific CS rates offered more accurate survival information for patients who have already survived a certain period of time after diagnosis.
\end{abstract}

Keywords: Gallbladder cancer, Conditional survival, SEER

\section{Introduction}

Although gallbladder (GB) cancer is not a common malignancy with approximately 5000 new cases per year in the United States, it is the most common biliary tract cancer and the fifth most common gastrointestinal malignancy [1]. The prognosis of GB cancer is poor, with a 5-year overall survival (OS) rate of 5\%-17\% [2-5]. North Indians [6] and South American Indians [7] have a high incidence of GB cancer with a worse prognosis, as the 5 -year survival rate is reported to be less than $10 \%$.

\footnotetext{
*Correspondence: kyubokim.ro@gmail.com

Department of Radiation Oncology, Ewha Womans University College of Medicine, 1071 Anyangcheon-ro, Yangcheon-gu, Seoul 07985, Republic of Korea
}

However, more aggressive surgery and the use of adjuvant therapy have improved survival outcomes over the last few decades [8-11].

Prognostic estimation for cancer patients is usually performed using the cancer-specific survival (CSS) measurement. As the CSS rate is defined as the survival probability from diagnosis to a specific time point, CSS underestimates the actual survival rates of the cancer survivors who have already survived a certain period. This underestimation is prominent in cancer patients with poor CSS such as GB cancer patients.

Cancer-specific conditional survival (CS) rate is defined as the probability that a cancer patient will survive some additional number of years, given the condition that 
the patient has already survived for a certain number of years. In actual clinical practice, the cancer-specific CS rate is informative for patients and their clinicians because the cancer-specific CS rate applies the condition that the patient is still alive [12]. Incorporating information on alive status generates more relevant survival estimation (CS rate) than the classical CSS rate [13]. Therefore, the use of cancer-specific CS rate is meaningful during patient counseling.

Furthermore, the cancer-specific CS rate can be used as a surrogate for cure rate. If the cancer-specific CS rate reaches a plateau (i.e., ceiling) at a certain time point, a patient who survives to the time point with no evidence of disease can be considered to be cured [14]. Analyzing the cancer-specific CS rate of GB cancer may provide quantitative insight into the curability of GB cancer.

In this study, we calculated the cancer-specific CS rate of GB cancer using the Surveillance, Epidemiology, and End Results (SEER) database between 2004 and 2013. The over-time changes of prognostic significance of patient, tumor, and treatment-related factors were analyzed.

\section{Methods and materials Patient population}

The SEER 18-registry dataset (a set of 18 populationbased regional cancer registries) was used in this study. Patients who were pathologically or clinically diagnosed with primary GB cancer (ICD-0-3 code 23.9/WHO 2008) between 2004 and 2013 were identified. The patients with unknown survival time were excluded. Tumors were classified according to the 7th edition of the American Joint Committee on Cancer (AJCC) staging manual [15].

\section{Statistical analysis}

The formula of cancer-specific CS rate is as follows [12]; $\operatorname{CSS}(t)$ is the $t$-year CSS rate. Cancer-specific $\operatorname{CS}(y \mid x)$ is the additional $y$-year CSS rate, given the condition that the person has already survived $x$ years.

$$
\text { cancer }- \text { specific } \operatorname{CS}(y \mid x)=\frac{\operatorname{CSS}(x+y)}{\operatorname{CSS}(x)}
$$

For example, to calculate the 3-year cancer-specific CS rate for a patient who has already survived 2 years $(x=2, y=3)$, the 5 -year CSS rate, CSS $(2+3)$, is divided by the 2-year CSS rate, CSS(2). Suppose that there were 100 patients diagnosed with GB cancer. Among them, 50 patients have survived from the cancer for 2 years $[\operatorname{CSS}(2)=0.5]$, and 20 patients have survived for 5 years $[\mathrm{CSS}(5)=0.2]$. In that case, the 3 -year cancer-specific CS rate at 2 years after diagnosis is $0.4(0.2 / 0.5)$.

$$
\text { cancer }- \text { specific } \operatorname{CS}(3 \mid 2)=\frac{\operatorname{CSS}(2+3)}{\operatorname{CSS}(2)}=\frac{\frac{20}{100}}{\frac{50}{100}}=\frac{20}{50}=0.4
$$

In this formula, the initial settings of 100 patients and the 2-year time interval are eliminated. Cancer-specific $\mathrm{CS}(3 \mid 2)$ can be defined as the 3-year CSS rate of the selective patients who survived for 2 years $(n=50)$ calculated by using newly formatted survival time (subtraction of 2 years from the initial survival time).

With this concept, the 3-year cancer-specific CS rates for the patients who survived for $x$ years were computed by following procedures: (1) selection of $x$-year survivors; (2) subtraction of $x$ years from the initial survival time; and (3) calculation of a 3-year CSS rate for the survivors using the modified survival time.

The Kaplan-Meier method was used to estimate the CSS and cancer-specific CS rates. To calculate $95 \%$ confidence intervals (CIs) of the CSS and cancer-specific CS rates, the $\log -\log$ transformation of survival was used [16].

Multivariate Cox proportional-hazards regression was performed to evaluate the hazard of CSS rate at the time of diagnosis and cancer-specific CS rates for multiple survival periods ( 1,3 , and 5 years after diagnosis). For instance, to compute the cancer-specific CS rate at 1 year after diagnosis, 1-year survivors were selected. After subtraction of 12 months from their survival time, a multivariate analysis was performed. Incorporated variables for the analysis at diagnosis were demographic (age at diagnosis, sex, race, marital status, insurance status), tumor (histology, grade, T, N, and M categories), and treatment-related factors (surgical extent, radiotherapy). Only the variables which were prognostic with $P$ value less than 0.1 in the analysis of the previous period were selected and incorporated in the next period's multivariate analysis sequentially. The multivariate Cox proportional hazards regression was performed using SPSS version 22.0 (SPSS Inc., Chicago, IL, USA).

At the same time, considering the potential influence of covariates on the survival at each time point, the covariate-adjusted survival function in the Cox model was used to estimate the adjusted CSS and cancer-specific CS rates. Specifically, the CSS and cancer-specific CS rates calculated with an adjustment for age, sex, race, histology, grade, $\mathrm{T}, \mathrm{N}$, and $\mathrm{M}$ categories, surgery, radiotherapy, insurance, and marital status. The log-log-based point-wise CIs were obtained for the adjusted CSS and cancer-specific CS rates [17]. For example, to calculate the adjusted 3-year cancer-specific CS rate at 1 year after diagnosis, the patients who have survived 12 months were selected and these 12 months were subtracted from the survival times of the survivors. Subsequent analysis was performed using the Cox regression while incorporating all variables. Given the estimated coefficients from the Cox model, a covariate-adjusted survival function estimate was performed, and the estimated survival 
rate at 36 months (that is, 48 months from diagnosis) was obtained as the adjusted 3-year cancer-specific CS rate at 1 year after diagnosis.

For subgroup analyses, the variables, which were found to be significant prognostic factors in the multivariate analyses, were selected to divide patients into multiple risk groups. The adjusted CSS rates and 3-year cancer-specific CS rates at 1, 2, 3, 4, and 5 years after diagnosis were calculated and compared among risk groups.

All calculations of unadjusted or adjusted CSS and cancer-specific CS rates were carried out using STATA/MP (ver. 14.2; StataCorp LP, College Station, TX, USA).

\section{Results}

\section{Demographic and clinicopathologic characteristics}

A total of 9760 patients diagnosed with GB cancer between 2004 and 2013 were included in our analyses. The number of patients who were still alive was 3232, 1178 , and 612 at 1,3 , and 5 years after diagnosis, respectively. Table 1 shows the characteristics of the patients at diagnosis as well as at 1,3 , and 5 years after diagnosis.

At diagnosis, the majority of patients were women $(n=6748,69.1 \%)$ and white $(n=7528,77.1 \%)$. A considerable proportion of patients had high-risk tumors with advanced T category (T3 or T4, $n=4492,46.0 \%$ ), lymph node involvement $(n=2623,26.9 \%)$, and distant metastasis $(n=3266,33.5 \%)$. A majority of patients $(n=6321$, $64.8 \%$ ) received surgical treatment at primary site. Radiotherapy was administered in $1254(12.8 \%)$ patients.

\section{Trends of CSS and adjusted CSS rates}

The overall CSS rate was 54.4, 40.2, 34.3, 31.6, and $29.6 \%$ at $1,2,3,4$, and 5 years, respectively. The covariate-adjusted CSS rate showed a similar trend as the unadjusted CSS rate, although that was slightly lower (54.6, 38.3, 31.3, 28.3, and $26.1 \%$ at $1,2,3,4$, and 5 years, respectively) (Fig. 1).

\section{Trends of CS and adjusted CS rates}

While the CSS rate decreased over time, the 3-year cancer-specific CS rate increased gradually in each successive year. The increased 3-year cancer-specific CS rate approached a plateau phase $(82.3,88.0$, and $92.3 \%$ at 3 , 4 , and 5 years, respectively), suggesting that the majority of survivors at 5 years after diagnosis achieved stable cures of their GB cancer (Fig. 1). Even after covariate adjustment, the cancer-specific CS rates increased over time to reach a plateau. Although the rates were slightly lower than the unadjusted rates, the adjusted cancer-specific CS rate reached to $90.5 \%$ at 5 years after diagnosis.

\section{Factors associated with CSS and cancer-specific CS rates}

Upon multivariate analysis at the time of diagnosis, patients who were young, white, had papillary histology, had low grade tumors, low T, N, and M categories, underwent surgery and radiotherapy, were insured, and were married had significantly higher CSS rates than the controls (Table 2). At 1 year after diagnosis, age 80 years or more $(P=0.007)$, black race $(P=0.040)$, high grade $(P=0.043)$, high $\mathrm{T}, \mathrm{N}$, and $\mathrm{M}$ categories $(P<0.001$ for all) were identified as significantly adverse predictors, and papillary histology $(P=0.003)$, surgery $(P=0.012)$, and radiotherapy $(P=0.015)$ were significantly favorable predictors. At 3 years after diagnosis, T3-4 and M1 diseases continued to predict low cancer-specific CS rate for survivors $(P<0.001$ and $P=0.038$, respectively). On multivariate analysis incorporating age, $\mathrm{T}$ and $\mathrm{M}$ categories, and surgery at 5 years after diagnosis, we found $\mathrm{T}$ category (for T2 disease, hazard ratio [HR] 1.346 and 95\% CI $0.567-3.199, P=0.501$; for T3-4 disease, HR 3.388 and 95\% CI 1.400-8.196; $P=0.007$ ) and M category (for M1, HR 4.620 and $95 \%$ CI 1.476-14.457; $P=0.009$ ) to be persistently significant prognostic factors.

\section{Subgroup analysis of CSS and cancer-specific CS rates}

The adjusted 5-year CSS rates of patients with T1, T2, and T3-4 disease were $56.8,31.6$, and $9.9 \%$, respectively. The adjusted 3-year cancer-specific CS rate for patients with T3-4 disease showed the greatest improvement from $13.9 \%$ at 1 year after diagnosis to $84.4 \%$ at 5 years after diagnosis. However, the significant inferiority of the 3 -year cancer-specific CS rate continued at 3 years after diagnosis. The 3 -year cancer-specific CS rates at 5 years for patients with T1, T2, and T3-4 disease were 94.0, 93.0, and $84.4 \%$, respectively, suggesting that the 5-year survivors with $\mathrm{T} 1-2$ diseases achieved stable cures of their disease.

The adjusted 5-year CSS rate of patients with distant metastasis (M1 disease) at time of diagnosis was only $2.5 \%$. The improvement of 3-year cancer-specific CS rate was decelerated and eventually ceased at $76.7 \%$ at 5 years after diagnosis, indicating that patients with M1 disease at diagnosis still experience disease progression despite surviving 5 years (Fig. 2).

Surgery increased the 5-year CSS rate from $8.8 \%$ to $37.3 \%$, with a 3 -year cancer-specific CS rate of $90.5 \%$ at 5 years after diagnosis. However, radiotherapy did not show any CSS and cancer-specific CS benefits. The patients who had low cancer-specific CS rates at an early period in the radiotherapy group were prone to have a low CSS rate at the late period. At 5 years after diagnosis, the CSS rates were $25.7 \%$ and $7.8 \%$ in the non-radiotherapy group and radiotherapy group, respectively. The 
Table 1 Characteristics of patients with gallbladder cancer at the time of diagnosis and survivor characteristics at 1, 3, and 5 years after diagnosis

\begin{tabular}{|c|c|c|c|c|}
\hline \multirow[t]{2}{*}{ Characteristic } & \multirow[t]{2}{*}{ At diagnosis $(n=9760)$} & \multicolumn{3}{|c|}{ Time after diagnosis } \\
\hline & & 1 year $(n=3232)$ & 3 years $(n=1178)$ & 5 years $(n=612)$ \\
\hline \multicolumn{5}{|c|}{ Age at diagnosis (years) } \\
\hline$<65$ & $3056(31.3)$ & $1223(37.8)$ & $470(39.9)$ & $251(41.0)$ \\
\hline $65-79$ & 3975 (40.7) & $1339(41.4)$ & $493(41.9)$ & $261(42.6)$ \\
\hline$\geq 80$ & $2729(28.0)$ & $670(20.7)$ & $215(18.3)$ & $100(16.3)$ \\
\hline \multicolumn{5}{|l|}{ Sex } \\
\hline Men & $3012(30.9)$ & $956(29.4)$ & $320(27.2)$ & $158(25.8)$ \\
\hline Women & $6748(69.1)$ & $2276(70.4)$ & $858(72.8)$ & $454(74.2)$ \\
\hline \multicolumn{5}{|l|}{ Race } \\
\hline White & $7528(77.1)$ & $2506(77.5)$ & $908(77.1)$ & $473(77.3)$ \\
\hline Black & $1163(11.9)$ & $370(11.4)$ & $128(10.9)$ & $64(10.5)$ \\
\hline Others & $1040(10.7)$ & $347(10.7)$ & $139(11.8)$ & $73(11.9)$ \\
\hline Unknown & $29(0.3)$ & $9(0.3)$ & $3(0.3)$ & $2(0.3)$ \\
\hline \multicolumn{5}{|l|}{ Histology } \\
\hline Non-papillary & $9415(96.5)$ & 3007 (93.0) & $1059(89.9)$ & $547(89.4)$ \\
\hline Papillary & $345(3.5)$ & $225(7.0)$ & $119(10.1)$ & $65(10.6)$ \\
\hline \multicolumn{5}{|l|}{ Grade } \\
\hline Grade 1 or 2 & $3648(37.4)$ & $1821(56.3)$ & $716(60.8)$ & $372(60.8)$ \\
\hline Grade 3 or 4 & $2874(29.4)$ & $790(24.4)$ & $243(20.6)$ & $121(19.8)$ \\
\hline Unknown & $3238(33.2)$ & $621(19.2)$ & $219(18.6)$ & $119(19.4)$ \\
\hline \multicolumn{5}{|l|}{ T category } \\
\hline $\mathrm{T} 1$ & $1520(15.6)$ & $817(25.3)$ & $433(36.8)$ & $257(42.0)$ \\
\hline $\mathrm{T} 2$ & 2354 (24.1) & $1276(39.5)$ & $504(42.8)$ & $241(39.4)$ \\
\hline T3-4 & $4492(46.0)$ & $979(30.3)$ & $212(18.0)$ & $99(16.2)$ \\
\hline Unknown & 1394 (14.3) & $160(5.0)$ & $29(2.5)$ & $15(2.5)$ \\
\hline \multicolumn{5}{|l|}{ N category } \\
\hline No & $5521(56.6)$ & $2220(68.7)$ & $927(78.7)$ & $495(80.9)$ \\
\hline $\mathrm{N} 1-2$ & 2623 (26.9) & $786(24.3)$ & $188(16.0)$ & $82(13.4)$ \\
\hline Unknown & $1616(16.6)$ & $226(7.0)$ & $63(5.3)$ & $35(5.7)$ \\
\hline \multicolumn{5}{|l|}{ M category } \\
\hline MO & 5594 (57.3) & 2665 (82.5) & $1085(92.1)$ & $568(92.8)$ \\
\hline M1 & 3266 (33.5) & 408 (12.6) & $40(3.4)$ & $16(2.6)$ \\
\hline Unknown & $900(9.2)$ & $159(4.9)$ & $53(4.5)$ & $28(4.6)$ \\
\hline \multicolumn{5}{|l|}{ Surgery } \\
\hline No & 3393 (34.8) & $348(10.8)$ & $55(4.7)$ & $32(5.2)$ \\
\hline Yes & $6321(64.8)$ & 2873 (88.9) & 1119 (95.0) & $578(94.4)$ \\
\hline Unknown & $46(0.5)$ & $11(0.3)$ & $4(0.3)$ & $2(0.3)$ \\
\hline \multicolumn{5}{|l|}{ Radiotherapy } \\
\hline No & 8329 (85.3) & 2483 (76.8) & 949 (80.6) & $505(82.5)$ \\
\hline Yes & 1254 (12.8) & $687(21.3)$ & $215(18.3)$ & $100(16.3)$ \\
\hline Unknown & $177(1.8)$ & $62(1.9)$ & $14(1.2)$ & $7(1.1)$ \\
\hline \multicolumn{5}{|l|}{ Insurance } \\
\hline No & $1484(15.2)$ & $436(13.5)$ & $134(11.4)$ & $47(7.7)$ \\
\hline Yes & $5311(54.4)$ & $1769(54.7)$ & $547(46.4)$ & $204(33.3)$ \\
\hline Unknown & 2965 (30.4) & 1027 (31.8) & $497(42.2)$ & 361 (59.0) \\
\hline
\end{tabular}


Table 1 continued

\begin{tabular}{|c|c|c|c|c|}
\hline \multirow[t]{2}{*}{ Characteristic } & \multirow[t]{2}{*}{ At diagnosis $(n=9760)$} & \multicolumn{3}{|c|}{ Time after diagnosis } \\
\hline & & 1 year $(n=3232)$ & 3 years $(n=1178)$ & 5 years $(n=612)$ \\
\hline \multicolumn{5}{|l|}{ Marriage } \\
\hline No & $4610(47.2)$ & $1365(42.2)$ & $480(40.7)$ & $237(38.7)$ \\
\hline Yes & $4689(48.0)$ & $1723(53.3)$ & $651(55.3)$ & $346(56.5)$ \\
\hline Unknown & $461(4.7)$ & $144(4.5)$ & $47(4.0)$ & $29(4.7)$ \\
\hline
\end{tabular}

All values are presented as number of cases followed by percentage in parentheses

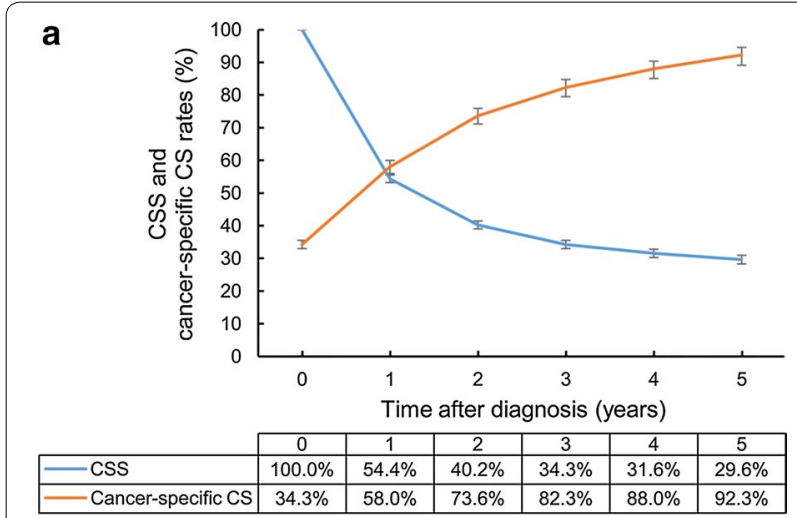

b

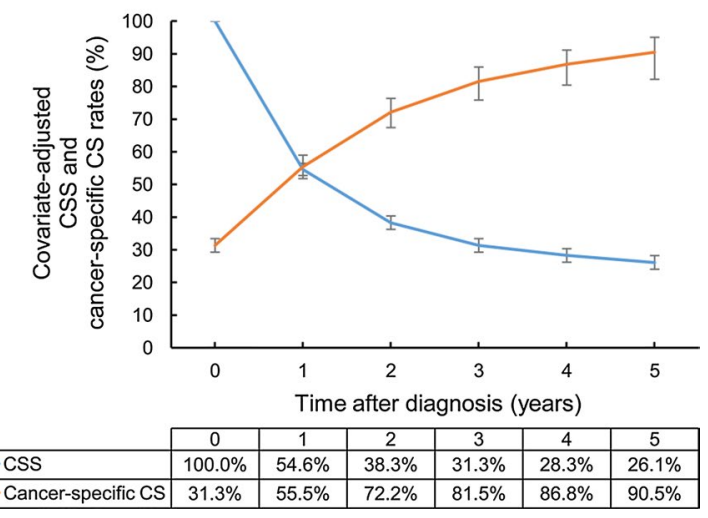

Fig. 1 CSS and 3-year cancer-specific CS rates of gallbladder cancer patients: a unadjusted (Kaplan-Meier) and $\mathbf{b}$ covariate-adjusted (Cox model) CSS and CS rates. Error bars indicate 95\% confidence intervals (Cls). CSS cancer-specific survival, CS conditional survival

adjusted 3-year cancer-specific CS rate at 5 years after diagnosis in the radiotherapy group was $82.5 \%$ (Fig. 3 ).

In subgroup analyses for patients treated with surgery, $\mathrm{T}$ and $\mathrm{M}$ categories were still significant prognostic factors. The 5-year CSS rates of patients with T1, T2, and T3-4 diseases were $65.7,48.9$, and $14.9 \%$, respectively, and the patients with $\mathrm{T} 1$ and $\mathrm{T} 2$ diseases had 3-year cancer-specific CS rates of higher than $90 \%$ at 5 years after diagnosis ( $94.2 \%$ and $92.5 \%$, respectively), whereas those with T3-4 diseases did not (78.6\%). Even after surgery, patients with M1 disease showed a low 5-year CSS rate $(7.1 \%$ vs. $45.5 \%)$ and 3-year cancer-specific CS rate $(42.8 \%$ vs. $91.6 \%)$ at 5 years after diagnosis compared with patients with M0 disease.

When radiotherapy was administered after surgery, the adjusted CSS rate at 1 year after diagnosis increased from $65.0 \%$ to $77.3 \%$. However, radiotherapy decreased the 3-year cancer-specific CS rate at an early period, resulting in a 5 -year CSS rate of $34.1 \%$ in the radiotherapy group, which was similar to $38.7 \%$ in the non-radiotherapy group (Fig. 4).

\section{Discussion}

In the present study, we found that the 3-year cancerspecific CS rate of GB cancer patients increased over time, resulting in the covariate-adjusted 3-year cancerspecific CS rate of $90.5 \%$ at 5 years after diagnosis. The covariate-adjusted 5-year CSS rate was $26.1 \%$. Therefore, $26.1 \%$ of GB cancer patients who survived 5 years can be expected to survive 3 more years with $90.5 \%$ of probability. $\mathrm{T}$ and $\mathrm{M}$ categories were significant prognostic factors at the time of diagnosis and their prognostic effects persisted until 5 years after diagnosis. However, local treatments at the time of diagnosis including surgery and radiotherapy were not prognostic factors at 5 years after diagnosis. The combined information of CSS and cancerspecific CS rates allowed more efficient prognostic and predictive analyses.

The CS rate is higher than the CSS rate estimated at the time of diagnosis. If a patient is still alive 2 years after diagnosis, the 3 -year CS rate at 2 years after diagnosis is higher than the 5-year CSS rate, because the 5-year CSS rate includes the probability of patients who died within 2 years after diagnosis. For patients who survive, the CS rate can offer more accurate information regarding survival estimation compared with the traditionally used CSS rate. The cancer-specific CS rate is usually higher than the CSS rate even in patients who are alive with disease, and the difference between both rates is even more distinct for patients with poor prognosis cancers [18]. Therefore, the cancer-specific CS rate is a more relevant prognostic factor compared with the CSS rate, especially for the survivors of poor prognosis cancers. 


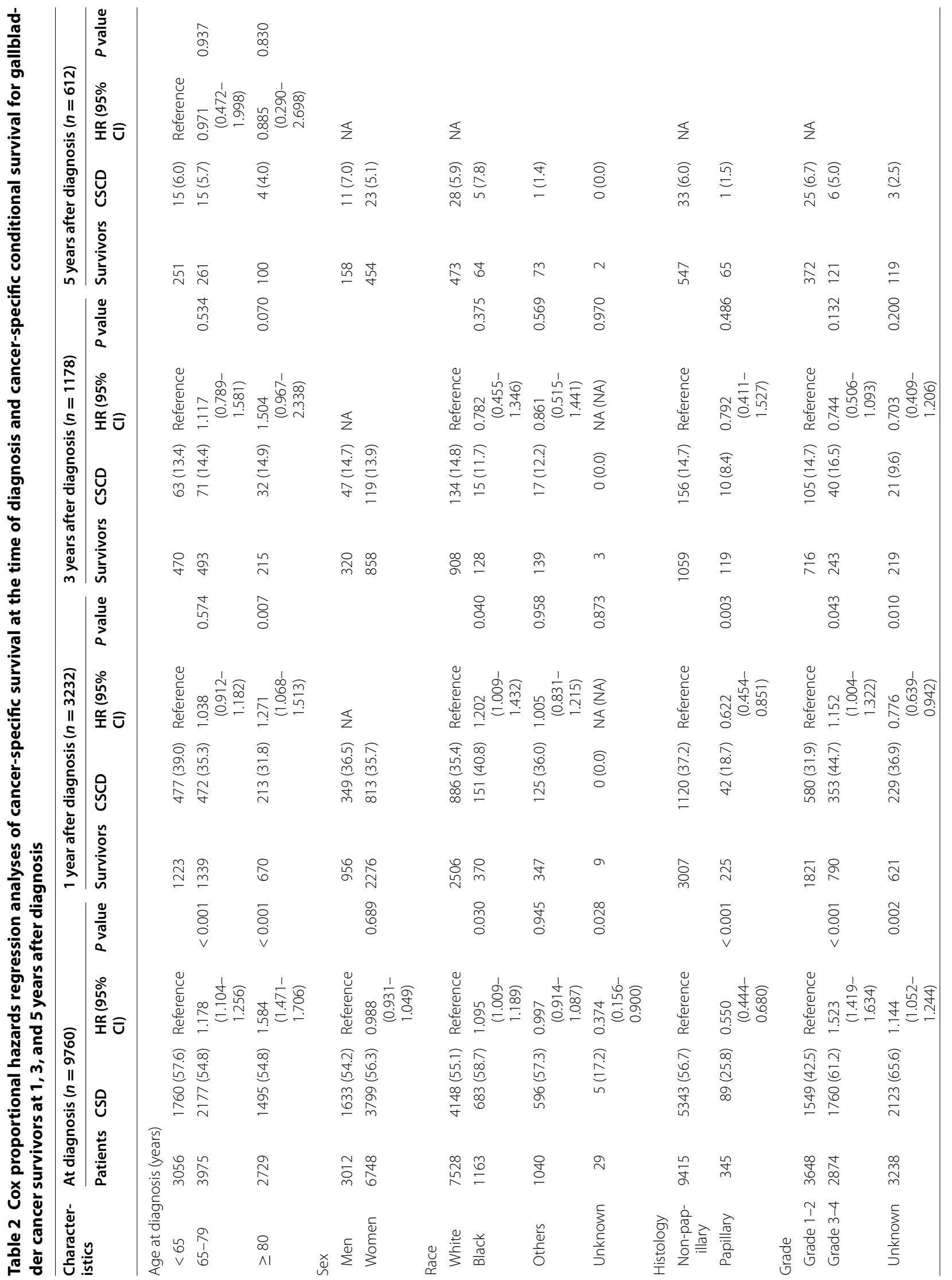




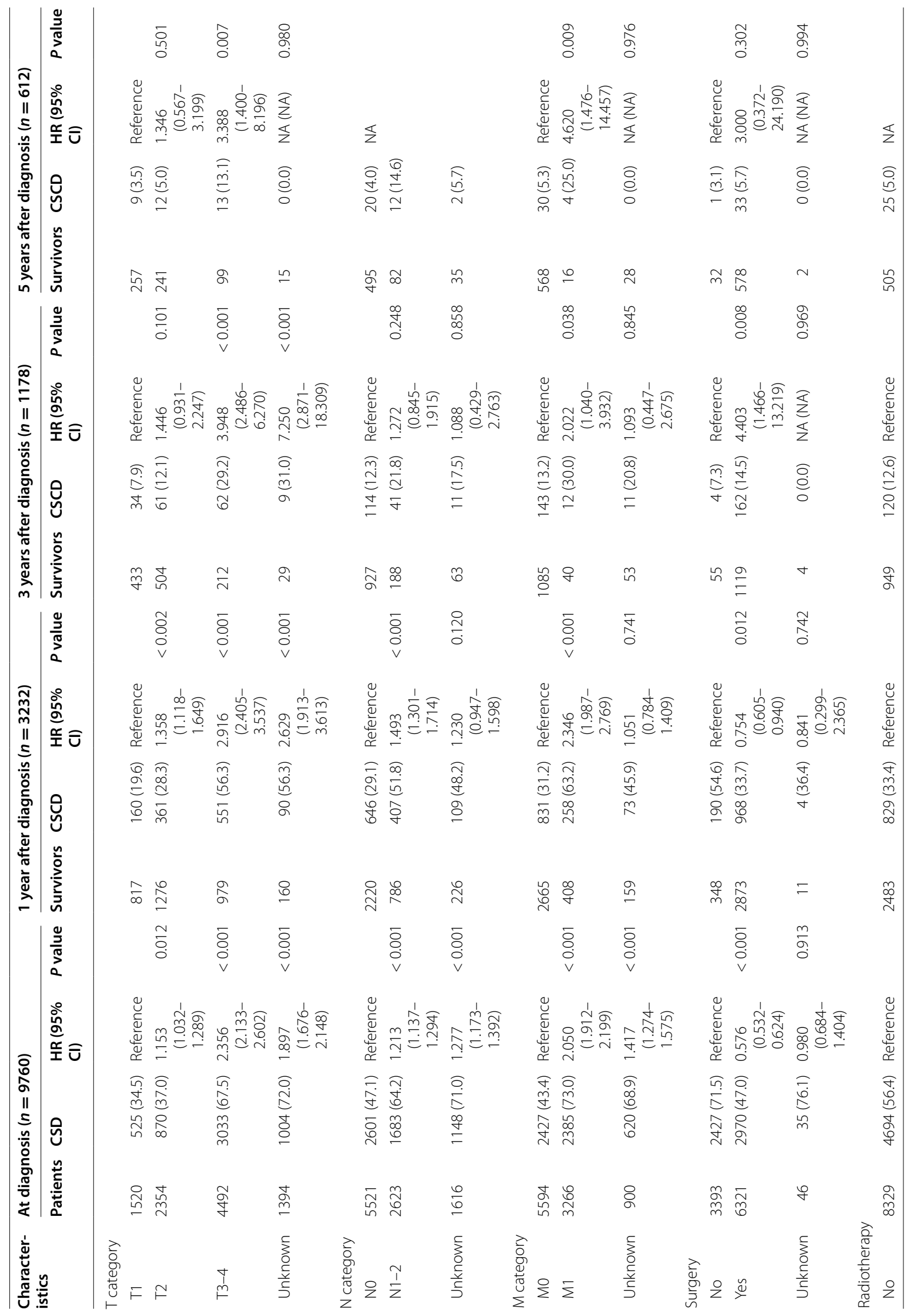




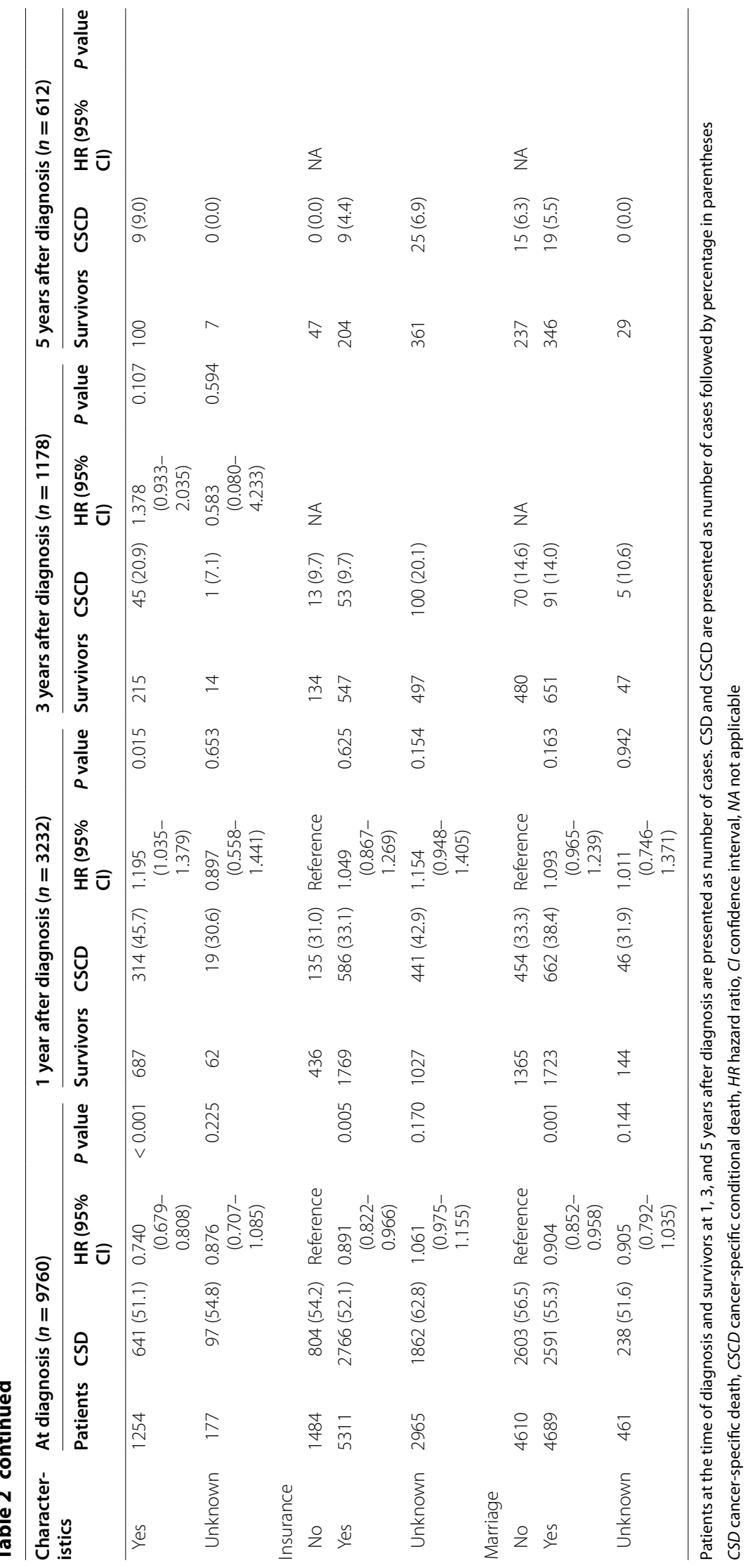



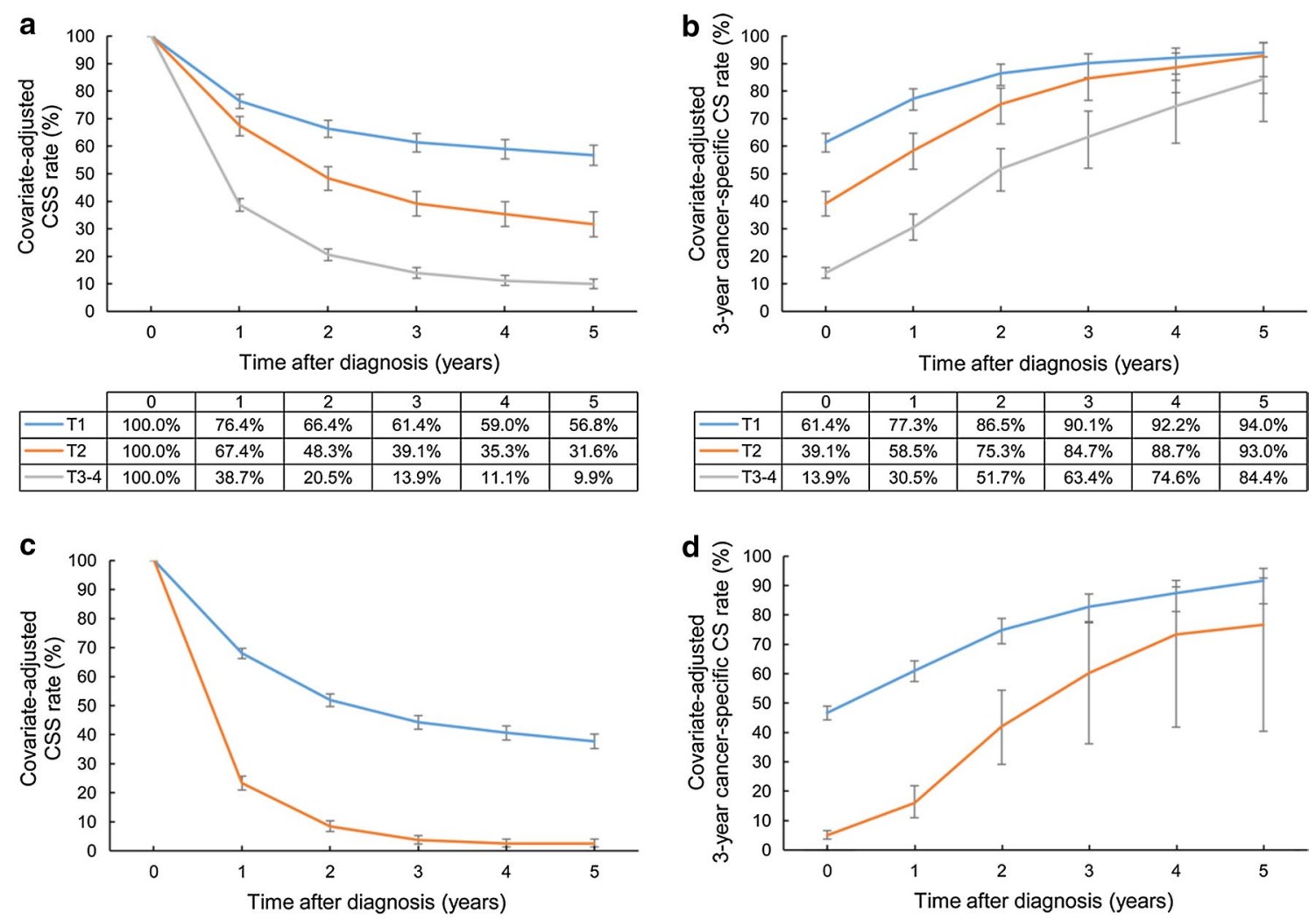

\begin{tabular}{|l|c|c|c|c|c|c|}
\cline { 2 - 7 } \multicolumn{1}{c|}{} & 0 & 1 & 2 & 3 & 4 & 5 \\
\hline - T1 & $61.4 \%$ & $77.3 \%$ & $86.5 \%$ & $90.1 \%$ & $92.2 \%$ & $94.0 \%$ \\
\hline - 2 2 & $39.1 \%$ & $58.5 \%$ & $75.3 \%$ & $84.7 \%$ & $88.7 \%$ & $93.0 \%$ \\
\hline- T3-4 & $13.9 \%$ & $30.5 \%$ & $51.7 \%$ & $63.4 \%$ & $74.6 \%$ & $84.4 \%$ \\
\hline
\end{tabular}

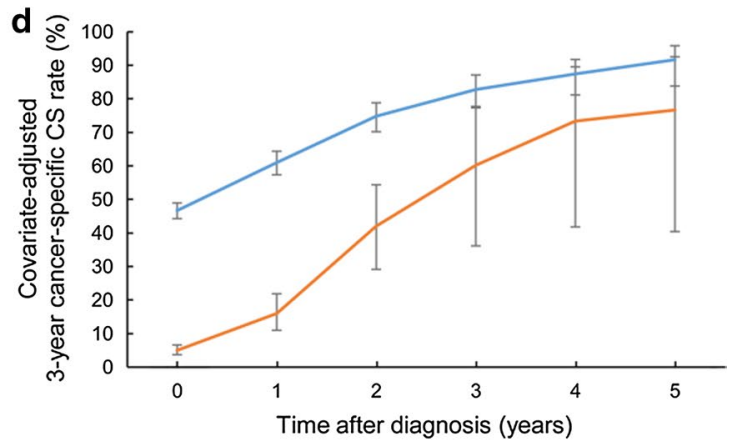

\begin{tabular}{|c|c|c|c|c|c|c|}
\cline { 2 - 7 } \multicolumn{1}{c|}{} & 0 & 1 & 2 & 3 & 4 & 5 \\
\hline$-\mathrm{M0}$ & $100.0 \%$ & $68.0 \%$ & $51.9 \%$ & $44.3 \%$ & $40.6 \%$ & $37.7 \%$ \\
\hline$-\mathrm{M} 1$ & $100.0 \%$ & $23.3 \%$ & $8.4 \%$ & $3.7 \%$ & $2.5 \%$ & $2.5 \%$ \\
\hline
\end{tabular}

\begin{tabular}{|c|c|c|c|c|c|c|}
\cline { 2 - 7 } \multicolumn{1}{c|}{} & 0 & 1 & 2 & 3 & 4 & 5 \\
\hline$-\mathrm{M} 0$ & $46.6 \%$ & $61.0 \%$ & $74.8 \%$ & $82.8 \%$ & $87.5 \%$ & $91.7 \%$ \\
\hline$-\mathrm{M} 1$ & $5.0 \%$ & $16.0 \%$ & $42.0 \%$ & $60.3 \%$ & $73.3 \%$ & $76.7 \%$ \\
\hline
\end{tabular}

Fig. 2 Covariate-adjusted (Cox model) CSS and 3-year cancer-specific CS rates of gallbladder cancer patients according to T and M categories: a CSS rates according to T category; b cancer-specific CS rates according to T category; $\mathbf{c}$ CSS rates according to M category; $\mathbf{d}$ cancer-specific CS rates according to M category. Error bars indicate 95\% Cls. CSS cancer-specific survival, CS conditional survival

One of the advantages of the CS model compared with the Kaplan-Meier survival curve or multivariate regression models is that it offers more intuitive and quantitative information about the cure rate. Even without information about cancer recurrence or progression, the percentage of remaining patients when the CS rate reaches to a plateau (e.g., > 90\%) could be used as a surrogate for the stable cure rate of the disease [14]. By using CS analysis, the benefit of treatments can also be evaluated; the difference of the percentage of patients who reach a plateau of CS rate between control and treatment groups can be considered the improved cure rate from the treatment [19-24].

In the present study, multivariate analyses of cancerspecific CS rates at 1,3 , and 5 years after diagnosis also provided useful information that $\mathrm{T}$ and $\mathrm{M}$ categories were the most significant prognostic factors even at 5 years after diagnosis, whereas surgery and radiotherapy were not. This finding suggests that patients with high risks might benefit from adjuvant treatments including systemic therapy after initial radical treatments.
There is no argument based on our analysis that complete resection with negative margin is a necessary condition for potentially curative treatment, whereas the role of both chemotherapy and radiotherapy have not been fully established [25-27]. In our present study, surgery increased the 5 -year CSS rate to $28.5 \%$ with a 3 -year cancer-specific CS rate of $90.5 \%$ at 5 years after diagnosis. However, radiotherapy did not increase CSS or CS rates at 5 years after diagnosis even after covariate adjustment. In the subgroup analysis of the surgery group, although radiotherapy increased the 1-year CSS rate, the lower cancer-specific CS rate at 1 year after diagnosis compared with the non-radiotherapy group indicated a poor prognosis at late period, resulting in no difference in the CSS rate at 5 years after diagnosis between the radiotherapy and non-radiotherapy groups.

Hyder et al. [28] demonstrated a similar conclusion in a propensity score-matched SEER data analysis that adjuvant external beam radiotherapy after curativeintent resection for GB cancer showed a survival benefit at 1 year after diagnosis and the benefit dissipated at 
a

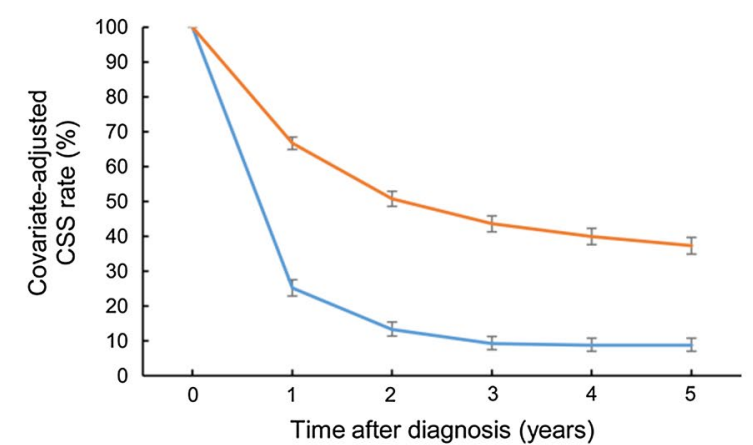

\begin{tabular}{|l|c|c|c|c|c|c|}
\cline { 2 - 7 } \multicolumn{1}{c|}{} & 0 & 1 & 2 & 3 & 4 & 5 \\
\hline - No surgery & $100.0 \%$ & $25.2 \%$ & $13.3 \%$ & $9.3 \%$ & $8.8 \%$ & $8.8 \%$ \\
\hline 一 Surgery & $100.0 \%$ & $66.7 \%$ & $50.8 \%$ & $43.6 \%$ & $39.9 \%$ & $37.3 \%$ \\
\hline
\end{tabular}

C

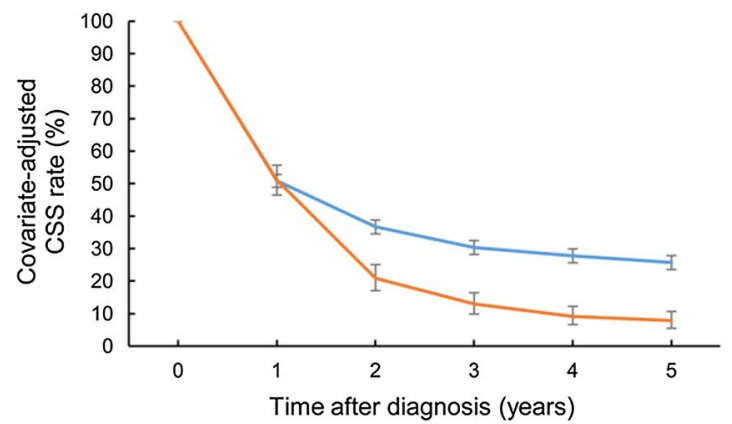

\begin{tabular}{|c|c|c|c|c|c|c|}
\cline { 2 - 7 } \multicolumn{1}{c|}{} & 0 & 1 & 2 & 3 & 4 & 5 \\
\hline No radiotherapy & $100.0 \%$ & $50.9 \%$ & $36.7 \%$ & $30.3 \%$ & $27.7 \%$ & $25.7 \%$ \\
\hline - Radiotherapy & $100.0 \%$ & $51.2 \%$ & $20.9 \%$ & $12.9 \%$ & $9.2 \%$ & $7.8 \%$ \\
\hline
\end{tabular}

Fig. 3 Covariate-adjusted (Cox model) CSS and 3-year cancer-specific CS rates of gallbladder cancer patients according to treatments: a CSS rates according to surgery; $\mathbf{b}$ cancer-specific CS rates according to surgery; $\mathbf{c}$ CSS rates according to radiotherapy; $\mathbf{d}$ cancer-specific CS rates according to

radiotherapy. Error bars indicate $95 \%$ Cls. CSS cancer-specific survival, CS conditional survival

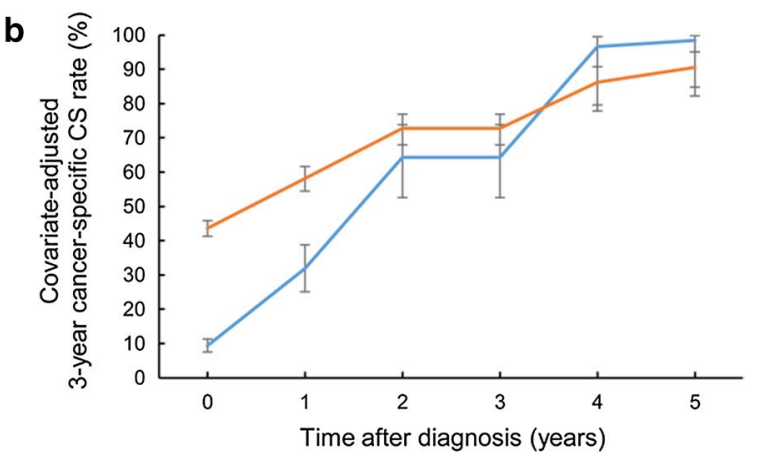

\begin{tabular}{|c|c|c|c|c|c|c|}
\cline { 2 - 7 } \multicolumn{1}{c|}{} & 0 & 1 & 2 & 3 & 4 & 5 \\
\hline - No surgery & $9.3 \%$ & $31.8 \%$ & $64.3 \%$ & $64.3 \%$ & $96.6 \%$ & $98.5 \%$ \\
\hline - Surgery & $43.6 \%$ & $58.2 \%$ & $72.7 \%$ & $72.7 \%$ & $86.2 \%$ & $90.5 \%$ \\
\hline
\end{tabular}

d

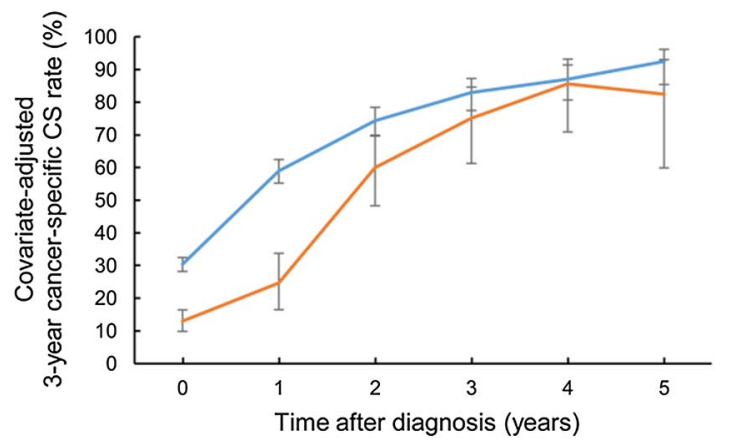

\begin{tabular}{|c|c|c|c|c|c|c|}
\cline { 2 - 7 } \multicolumn{1}{c|}{} & 0 & 1 & 2 & 3 & 4 & 5 \\
\hline - No radiotherapy & $30.3 \%$ & $58.9 \%$ & $74.4 \%$ & $83.0 \%$ & $87.0 \%$ & $92.5 \%$ \\
\hline - Radiotherapy & $12.9 \%$ & $24.7 \%$ & $60.0 \%$ & $75.1 \%$ & $85.6 \%$ & $82.5 \%$ \\
\hline
\end{tabular}

5 years after diagnosis. This result gives an impression that although radiotherapy may delay the progression of the disease, it can not increase long-term overall survival rate.

However, these results should be interpreted with caution. The discordance between the increased CSS rate and decreased cancer-specific CS rate at 1 year after diagnosis implies survivorship bias; patients who have benefit from radiotherapy might be those at high risk. In spite of the survival benefit of radiotherapy, the survival of the patients at high risk may be shorter than that of the patients at low risk, resulting in the change of the patient cohorts in the late period. Even in the study of propensity score-matched analysis of Hyder et al. [28], the patients with lymph node involvement had a longterm survival benefit from radiotherapy. Wang et al. [5, 29] built nomograms from the SEER database to predict the individualized survival benefit of adjuvant radiotherapy or chemoradiotherapy for patients with resected GB cancer, and these nomograms indicated that as least patients with $\mathrm{T} 2$ or $\mathrm{N} 1$ disease will gain survival benefit from radiotherapy.

One possible limitation of this study is that the consecutively decreasing number of patients in these time series multivariate analyses might have an influence on statistical significance. However, the sample size at 5 years after diagnosis was more than 600 for the analysis of the five variables, suggesting the impact would be minor.

If most of the patients with a specific adverse feature died during the early period, the cancer-specific CS rate would be less meaningful. For example, the estimated 1 -year survival rate of patients in the non-surgery group was only $25.2 \%$. In that case, a high cancer-specific CS rate at 5 years after diagnosis cannot be translated automatically into useless value of surgical treatment. Moreover, the Cox hazard ratio regression at late period is less powerful because of an insufficiently large sample size of patients at high risk (e.g., those with N1-2 diseases). A small number of survivors at 5 years after diagnosis 
a

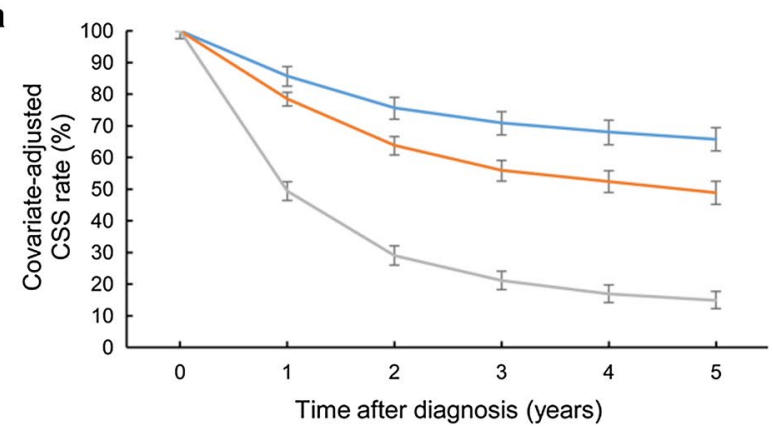

\begin{tabular}{|c|c|c|c|c|c|c|}
\cline { 2 - 7 } \multicolumn{1}{c|}{} & 0 & 1 & 2 & 3 & 4 & 5 \\
\hline - $100.0 \%$ & $85.8 \%$ & $75.7 \%$ & $71.0 \%$ & $68.1 \%$ & $65.7 \%$ \\
\hline \hline - 2 & $100.0 \%$ & $78.5 \%$ & $63.8 \%$ & $55.9 \%$ & $52.5 \%$ & $48.9 \%$ \\
\hline - $33-4$ & $100.0 \%$ & $49.4 \%$ & $29.0 \%$ & $21.1 \%$ & $16.9 \%$ & $14.9 \%$ \\
\hline
\end{tabular}

C

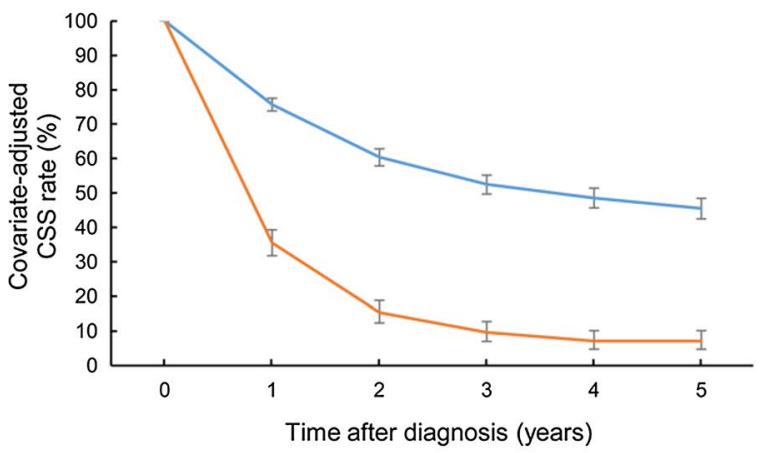

\begin{tabular}{|c|c|c|c|c|c|c|}
\cline { 2 - 7 } \multicolumn{1}{c|}{} & 0 & 1 & 2 & 3 & 4 & 5 \\
\hline \hline $\mathrm{M} 0$ & $100.0 \%$ & $75.7 \%$ & $60.4 \%$ & $52.5 \%$ & $48.6 \%$ & $45.5 \%$ \\
\hline \hline $\mathrm{M} 1$ & $100.0 \%$ & $35.5 \%$ & $15.4 \%$ & $9.6 \%$ & $7.1 \%$ & $7.1 \%$ \\
\hline
\end{tabular}

$\mathbf{e}$

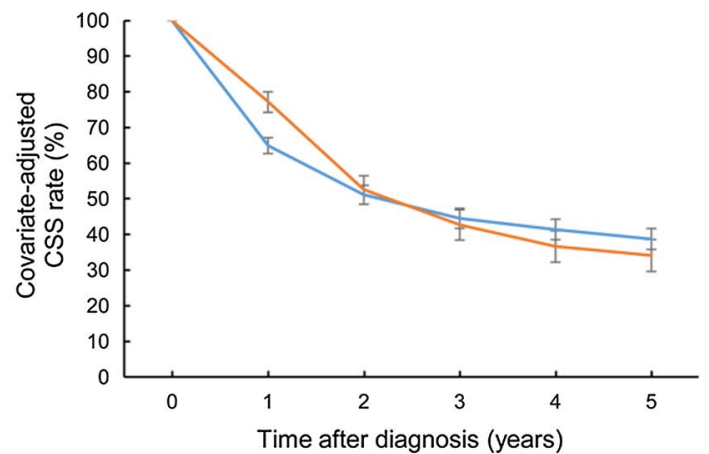

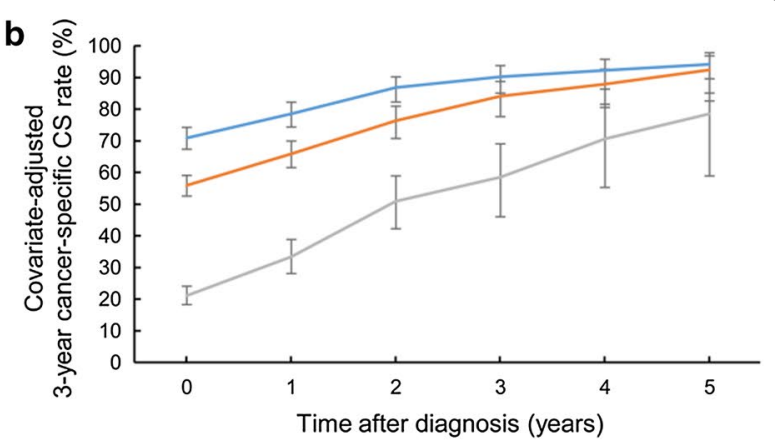

\begin{tabular}{|l|c|c|c|c|c|c|}
\cline { 2 - 7 } \multicolumn{1}{c|}{} & 0 & 1 & 2 & 3 & 4 & 5 \\
\hline$-\mathrm{T} 1$ & $71.0 \%$ & $78.6 \%$ & $86.8 \%$ & $90.3 \%$ & $92.3 \%$ & $94.2 \%$ \\
\hline$-\mathrm{T} 2$ & $55.9 \%$ & $65.9 \%$ & $76.3 \%$ & $84.1 \%$ & $87.9 \%$ & $92.5 \%$ \\
\hline$-\mathrm{T} 3-4$ & $21.1 \%$ & $33.4 \%$ & $50.9 \%$ & $58.5 \%$ & $70.7 \%$ & $78.6 \%$ \\
\hline
\end{tabular}

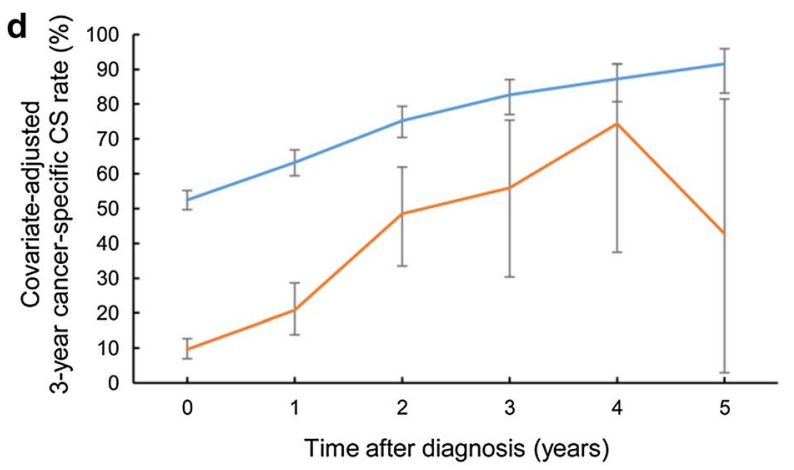

\begin{tabular}{|c|c|c|c|c|c|c|}
\cline { 2 - 7 } \multicolumn{1}{c|}{} & 0 & 1 & 2 & 3 & 4 & 5 \\
\hline$-\mathrm{M} 0$ & $52.5 \%$ & $63.3 \%$ & $75.2 \%$ & $82.7 \%$ & $87.2 \%$ & $91.6 \%$ \\
\hline$-\mathrm{M} 1$ & $9.6 \%$ & $20.7 \%$ & $48.5 \%$ & $56.0 \%$ & $74.4 \%$ & $42.8 \%$ \\
\hline
\end{tabular}

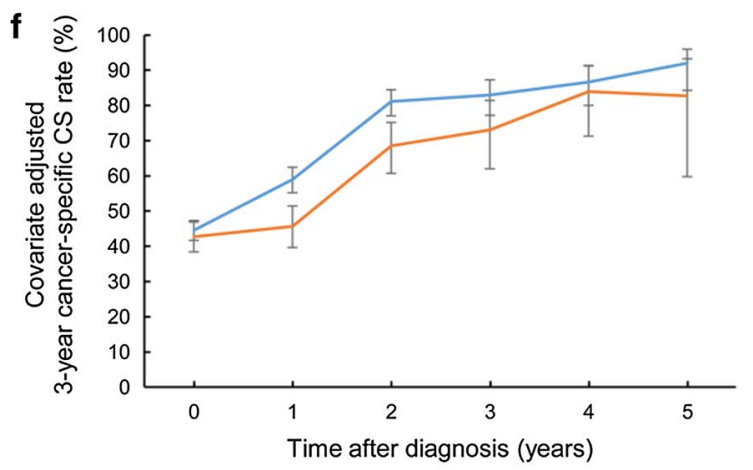

\begin{tabular}{|c|c|c|c|c|c|c|}
\cline { 2 - 7 } \multicolumn{1}{c|}{} & 0 & 1 & 2 & 3 & 4 & 5 \\
\hline - No radiotherapy & $44.5 \%$ & $58.9 \%$ & $81.1 \%$ & $82.9 \%$ & $86.7 \%$ & $92.0 \%$ \\
\hline - Radiotherapy & $42.7 \%$ & $45.7 \%$ & $68.6 \%$ & $73.1 \%$ & $84.0 \%$ & $82.7 \%$ \\
\hline
\end{tabular}

Fig. 4 Covariate-adjusted (Cox model) CSS and 3-year cancer-specific CS rates of gallbladder cancer patients treated with surgery: a CSS rates according to T category; $\mathbf{b}$ cancer-specific CS rates according to T category; c CSS rates according to M category; $\mathbf{d}$ cancer-specific CS rates according to $\mathrm{M}$ category; e rates CSS according to radiotherapy; $\mathbf{f}$ cancer-specific CS rates according to radiotherapy. Error bars indicate $95 \%$ Cls. CSS cancer-specific survival, CS conditional survival

reflects the low 5-year CSS rate in a risk group, whereas the statistical power of the cancer-specific CS rate at 5 years after diagnosis might be weakened by this small sample size at 5 years after diagnosis.
Jaundice and abnormal liver function could affect the patients' prognosis, and the presence of gallstone(s) is also a major risk factor for GB cancer [30-32]. However, these statuses were not included in the SEER database. 
SEER data did not offer any information on tumor recurrence or progression, surgical resection margin, and chemotherapy. With the data of patients with tumor recurrence, the conditional progression-free survival rate could be calculated and this rate would be more appropriate to serve as a surrogate for the cure rate of the patients with GB cancer [33].

\section{Conclusions}

The cancer-specific CS rate of patients with GB cancer offers more accurate survival information compared with the CSS rate to patients who survived for a certain period. $T$ and $M$ categories were still significant prognostic factors even 5 years after diagnosis, whereas local treatments at the time of diagnosis were not, suggesting that further adjuvant treatments might be helpful for the patients with high $\mathrm{T}$ and $\mathrm{M}$ categories.

\section{Authors' contributions}

KK conceived of the study, participated in its design and coordination, and revised the manuscript. YJK contributed to acquisition of data, statistical analyses, and writing. Both authors read and approved the final manuscript.

\section{Acknowledgements}

None.

\section{Competing interests}

The authors declare that they have no competing interests.

\section{Availability of data and materials}

The datasets generated and/or analyzed during the current study are available in the Surveillance, Epidemiology, and End Results Program repository, https:// seer.cancer.gov/seertrack/data/request/.

\section{Consent for publication}

Not applicable.

\section{Ethics approval and consent to participate}

Analysis of this de-identified dataset does not require Institutional Review Board approval.

\section{Funding}

None.

Received: 5 April 2017 Accepted: 19 October 2017

Published online: 30 October 2017

\section{References}

1. Baillie J. Tumors of the gallbladder and bile ducts. J Clin Gastroenterol. 1999;29(1):14-21.

2. Piehler J, Crichlow RW. Primary carcinoma of the gallbladder. Surg Gynecol Obstet. 1978;147(6):929-42.

3. Cubertafond P, Gainant A, Cucchiaro G. Surgical treatment of 724 carcinomas of the gallbladder. Results of the French Surgical Association Survey. Ann Surg. 1994;219(3):275-80.

4. Donohue JH, Nagorney DM, Grant CS, et al. Carcinoma of the gallbladder: does radical resection improve outcome? Arch Surg. 1990;125(2):237-41.

5. Wang SJ, Fuller CD, Kim J-S, et al. Prediction model for estimating the survival benefit of adjuvant radiotherapy for gallbladder cancer. J Clin Oncol. 2008;26(13):2112-7.
6. Kapoor VK, McMichael AJ. Gallbladder cancer: an 'Indian' disease. Natl Med J India. 2003;16(4):209-13.

7. Lazcano-Ponce EC, Miquel J, Muñoz N, et al. Epidemiology and molecular pathology of gallbladder cancer. CA Cancer J Clin. 2001;51(6):349-64.

8. Todoroki T, Kawamoto T, Takahashi H, et al. Treatment of gallbladder cancer by radical resection. Br J Surg. 1999;86(5):622-7.

9. Czito BG, Hurwitz HI, Clough RW, et al. Adjuvant external-beam radiotherapy with concurrent chemotherapy after resection of primary gallbladder carcinoma: a 23-year experience. Int J Radiat Oncol Biol Phys. 2005;62(4):1030-4.

10. Gold DG, Miller RC, Haddock MG, et al. Adjuvant therapy for gallbladder carcinoma: the Mayo clinic experience. Int J Radiat Oncol Biol Phys. 2009;75(1):150-5.

11. Bartlett DL, Fong Y, Fortner JG, et al. Long-term results after resection for gallbladder cancer. Implications for staging and management. Ann Surg. 1996;224(5):639-46.

12. Zabor EC, Gonen M, Chapman PB, et al. Dynamic prognostication using conditional survival estimates. Cancer. 2013;119(20):3589-92.

13. Choi $M$, Fuller $C D$, Thomas $C R$, et al. Conditional survival in ovarian cancer: results from the SEER dataset 1988-2001. Gynecol Oncol. 2008;109(2):203-9.

14. Chang GJ, Hu C-Y, Eng C, et al. Practical application of a calculator for conditional survival in colon cancer. J Clin Oncol. 2009;27(35):5938-43.

15. Edge SB, Compton CC. The American Joint Committee on Cancer: the 7th edition of the AJCC cancer staging manual and the future of TNM. Ann Surg Oncol. 2010;17(6):1471-4.

16. Cleves M. An introduction to survival analysis using stata. College Station: Stata Press; 2008.

17. Cefalu M. Pointwise confidence intervals for the covariate-adjusted survivor function in the cox model. Stata J. 2011;11(1):64-81.

18. Lv JW, Huang XD, Chen YP, et al. A national study of survival trends and conditional survival in nasopharyngeal carcinoma: analysis of the national population-based Surveillance Epidemiology and End Results registry. Cancer Res Treat. 2017. doi:10.4143/crt.2016.544 [Epub ahead of print]

19. Dikken JL, Baser RE, Gonen M, et al. Conditional probability of survival nomogram for 1-, 2-, and 3-year survivors after an $\mathrm{RO}$ resection for gastric cancer. Ann Surg Oncol. 2013;20(5):1623-30.

20. Karakiewicz Pl, Suardi N, Capitanio U, et al. Conditional survival predictions after nephrectomy for renal cell carcinoma. J Urol. 2009;182(6):2607-12.

21. Kang M, Kim HS, Jeong CW, et al. Conditional survival and associated prognostic factors in patients with upper tract urothelial carcinoma after radical nephroureterectomy: a retrospective study at a single institution. Cancer Res Treat. 2016;48(2):621-31.

22. Thuret $R$, Sun M, Abdollah F, et al. Conditional survival predictions after surgery for patients with penile carcinoma. Cancer. 2011;117(16):3723-30.

23. Spolverato G, Kim Y, Ejaz A, et al. Conditional probability of long-term survival after liver resection for intrahepatic cholangiocarcinoma: a multiinstitutional analysis of 535 patients. JAMA Surg. 2015;150(6):538-45.

24. Kim Y, Ejaz A, Spolverato G, et al. Conditional survival after surgical resection of gastric cancer: a multi-institutional analysis of the us gastric cancer collaborative. Ann Surg Oncol. 2015;22(2):557-64.

25. Shroff RT, Knox J, Dixon E. Consensus conference on gallbladder cancer. HPB. 2015;17(8):664-5.

26. Hueman MT, Vollmer CM Jr, PawlikTM. Evolving treatment strategies for gallbladder cancer. Ann Surg Oncol. 2009;16(8):2101-15.

27. Pilgrim CHC, Groeschl RT, Quebbeman EJ, et al. Recent advances in systemic therapies and radiotherapy for gallbladder cancer. Surg Oncol. 2013:22(1):61-7.

28. Hyder O, Dodson RM, Sachs T, et al. Impact of adjuvant external beam radiotherapy on survival in surgically resected gallbladder adenocarcinoma: a propensity score - matched Surveillance, Epidemiology, and End Results analysis. Surgery. 2014;155(1):85-93.

29. Wang SJ, Lemieux A, Kalpathy-Cramer J, et al. Nomogram for predicting the benefit of adjuvant chemoradiotherapy for resected gallbladder cancer. J Clin Oncol. 2011;29(35):4627-32. 
30. Hawkins WG, DeMatteo RP, Jarnagin WR, et al. Jaundice predicts advanced disease and early mortality in patients with gallbladder cancer. Ann Surg Oncol. 2004;11(3):310-5.

31. Andrea C, Enzo A. Cholesterol gallstones larger than $3 \mathrm{~cm}$ appear to be associated with gallbladder cancer: identification of a high risk group of patients that could benefit from preventive cholecystectomy. Ann Surg 2016;263(3):e56.
32. Hundal R, Shaffer EA. Gallbladder cancer: epidemiology and outcome. Clin Epidemiol. 2014;6:99-109.

33. Zamboni BA, Yothers G, Choi M, et al. Conditional survival and the choice of conditioning set for patients with colon cancer: an analysis of NSABP trials C-03 through C-07. J Clin Oncol. 2010;28(15):2544-8.

\section{Submit your next manuscript to BioMed Central and we will help you at every step:}

- We accept pre-submission inquiries

- Our selector tool helps you to find the most relevant journal

- We provide round the clock customer support

- Convenient online submission

- Thorough peer review

- Inclusion in PubMed and all major indexing services

- Maximum visibility for your research

Submit your manuscript at

www.biomedcentral com/submit 